\title{
Particle pair statistics of inertial particles at small separation using stereoscopic particle tracking
}

\author{
D. W. Hoffman*, J. K. Eaton \\ Stanford University, Department of Mechanical Engineering, Stanford, USA \\ *dwhoff@stanford.edu
}

\begin{abstract}
Particle pair statistics of inertial particles having average Stokes numbers of 2.1 and 14 are measured in isotropic turbulence at a Reynolds number of $R e_{\lambda}=240$. The radial distribution function (RDF) and mean relative approach velocity are obtained at small separation distances using 2-frame stereoscopic particle tracking velocimetry (stereo-PTV). At small separation distance, the RDF varies by an order of magnitude in the range of Stokes numbers investigated. However, the mean relative approach velocity is found to have a weak dependence on Stokes number. The results are shown to have high accuracy when compared to analogous mono-PTV datasets, and can be used to provide a more reliable estimate of the inter-particle collision rate. The main limitation of the measurement is observed at separation distances less than the laser sheet thickness, where the technique tended to underestimate the mean relative approach velocity.
\end{abstract}

\section{Introduction}

The agglomeration of inertial particles in turbulent flows takes place in a wide variety of settings, including rain formation, flocculation in water filtration, and volcanic ash dispersion. Agglomerate growth through inter-particle collisions can have a large impact on the transport of the particle phase, particularly when gravity acts to enhance sedimentation. The rate of aggregate growth is directly proportional to the particle collision frequency, which depends in part on the underlying carrier flow turbulence, particle time scale, and particle concentration. If the particle Stokes number based on the Kolmogorov time scale is moderate, the turbulence enhances the collision rate through the mechanism of preferential concentration (Eaton and Fessler, 1994). Sundaram and Collins (1997) were first to link the effects of preferential concentration to the volumetric collision rate $N_{\mathrm{c}}$ for spherical monodisperse particles with the following expression,

$$
N_{\mathrm{c}}=4 \pi n^{2} d_{\mathrm{p}}^{2} g\left(d_{\mathrm{p}}\right)\left\langle w_{\mathrm{r}}\right\rangle^{(-)}\left(d_{\mathrm{p}}\right),
$$

where $n$ is the particle number density, $d_{\mathrm{p}}$ is the particle diameter, $g(r)$ is the radial distribution function (RDF), and $\left\langle w_{\mathrm{r}}\right\rangle^{(-)}(r)$ is the mean relative approach velocity. Conventional 2D optical techniques can be used to measure $g(r)$ and $\left\langle w_{\mathrm{r}}\right\rangle^{(-)}(r)$ directly for $r>\delta z$, where $\delta z$ is the characteristic optical depth (eg. laser sheet thickness). However, it remains a challenge to obtain high accuracy for $d_{\mathrm{p}} \leq r \leq \delta z$ due to projection errors (Holtzer and Collins, 2002). Several studies have attempted to overcome these challenges using high resolution 3D particle tracking techniques. De Jong et al. (2010) used holographic imaging to obtain 3D particle coordinates and velocities, but the resulting mean approach velocity was heavily skewed by erroneous tails in the relative velocity distributions. Dou et al. (2018) extracted particle velocities with greater fidelity using 4-frame stereo particle tracking velocimetry (PTV). However, the extra optical components, instrumentation, and setup required to obtain meaningful results makes the method less attractive for numerous applications.

The focus of this paper is to evaluate a simpler 3D technique, 2-frame stereo-PTV, for the measurement of particle pair statistics at small separation in isotropic turbulence. We present the main limitations of the approach as well as recommendations for future improvements. 


\section{Experimental Methods}

Particle tracking measurements of inertial particles are carried out in a nearly isotropic turbulent flow. Particle coordinates and velocities are recorded as they undergo gravitational settling through the turbulence. The 2D and 3D coordinates are captured using a pair of stereoscopic cameras and a separate monoscopic camera respectively. Two types of particles are used, which exhibit different degrees of preferential concentration.

\subsection{Apparatus}

A turbulence tower, depicted in Fig. 11, is used to generate nearly isotropic turbulence in the absence of a net flow. Synthetic jets, powered by 4 inch acoustic woofers, are arranged along the walls of the tower, which has the shape of an octagonal prism. A thorough characterization of the turbulence statistics at the central axis of the tower is provided by Hoffman and Eaton (2021). The power used to actuate all synthetic jets combined is $29 \mathrm{~W}$, corresponding to a root-mean-square $(\mathrm{rms})$ velocity of $u^{\prime}=0.73 \mathrm{~m} / \mathrm{s}$, a dissipation rate of $\varepsilon=10.1 \mathrm{~m}^{2} / \mathrm{s}^{3}$, and a Reynolds number of $R e_{\lambda}=240$. A list of turbulence quantities is given in Table 1 , including the integral length scale $L$, Taylor microscale $\lambda_{f}$, Kolmogorov length scale $\eta$, and the Kolmogorov time scale $\tau_{\eta}$.

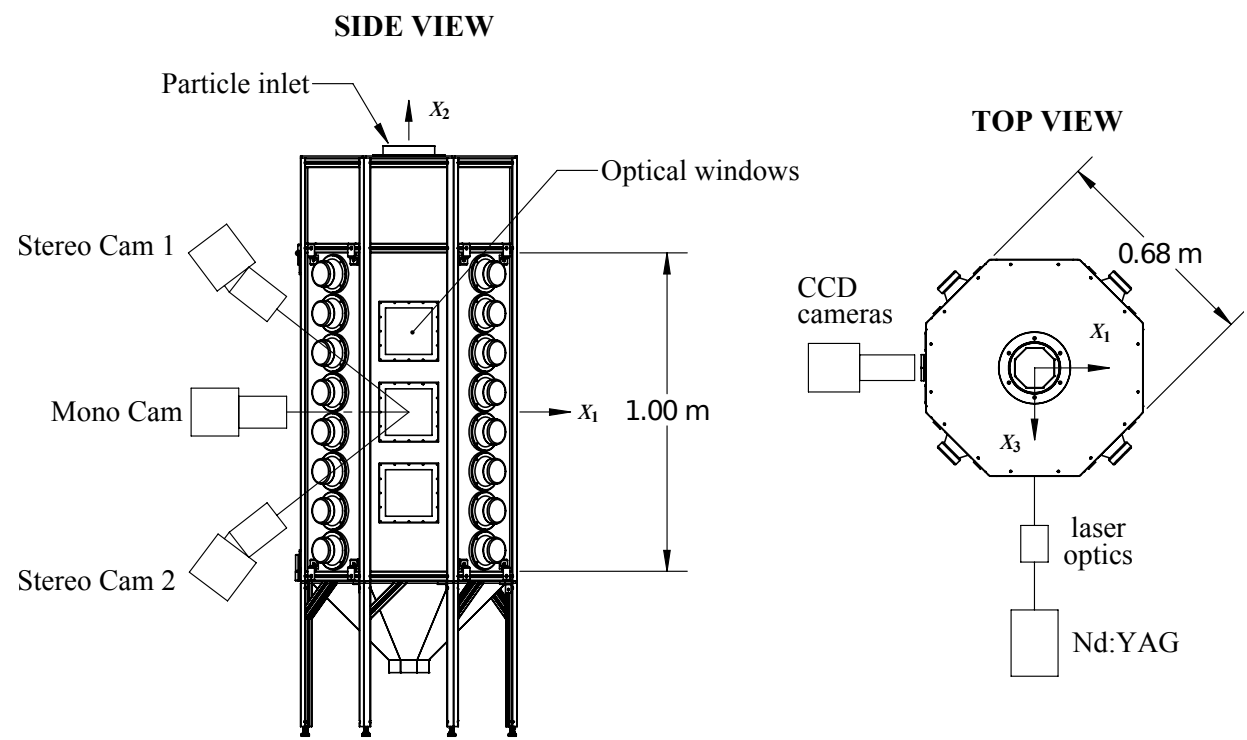

Figure 1: Turbulence tower used to generate nearly isotropic turbulence. Particles are introduced from the top and are illuminated on a plane passing through the central axis of the tower. Particle coordinates are recorded by independent mono and stereo camera setups.

Table 1: List of quantities characterizing the gas phase turbulence.

\begin{tabular}{rll}
\hline rms velocity & $u^{\prime}$ & $0.73 \mathrm{~m} / \mathrm{s}$ \\
integral length scale & $L$ & $71.9 \mathrm{~mm}$ \\
Taylor microscale & $\lambda_{f}$ & $4.9 \mathrm{~mm}$ \\
Reynolds number & $R e_{\lambda}=u^{\prime} \lambda_{f} / v$ & 240 \\
dissipation rate & $\varepsilon$ & $10.1 \mathrm{~m}^{2} / \mathrm{s}^{3}$ \\
Kolmogorov length scale & $\eta$ & $135 \mu \mathrm{m}$ \\
Kolmogorov time scale & $\tau_{\eta}$ & $1.2 \mathrm{~ms}$ \\
\hline
\end{tabular}


Particles are introduced into the turbulence tower from above. The particle feeding system (not shown in Fig. 1p consists of a hopper and a stack of dispersing sieves located above the tower. At the start of each experiment, particles are gravity fed through a small opening at the bottom of the hopper, dispersed through the sieves, and enter the tower. The turbulence fully disperses them throughout the test section. Due to the Basset history force, particles that enter the imaged volume from outside of the isotropic region can complicate the overall particle kinematics. Since the isotropic region extends above and below the measurement volume by about $0.5 \mathrm{~m}$, the history effects are likely small for particles with fast settling rates (i.e. on the order of the rms velocity fluctuations).

\subsection{Particle characterization}

Two types of glass microspheres, one solid and the other hollow, are studied. To obtain the size distribution, the solid glass particles are prepared in an electrolytic particle slurry and then measured with a Coulter counter. The resulting distribution of particle diameters is plotted in solid black in Fig. 2. The distribution is nearly monodisperse with a mean diameter of $53 \mu \mathrm{m}$ and a standard deviation of $4.0 \mu \mathrm{m}$.

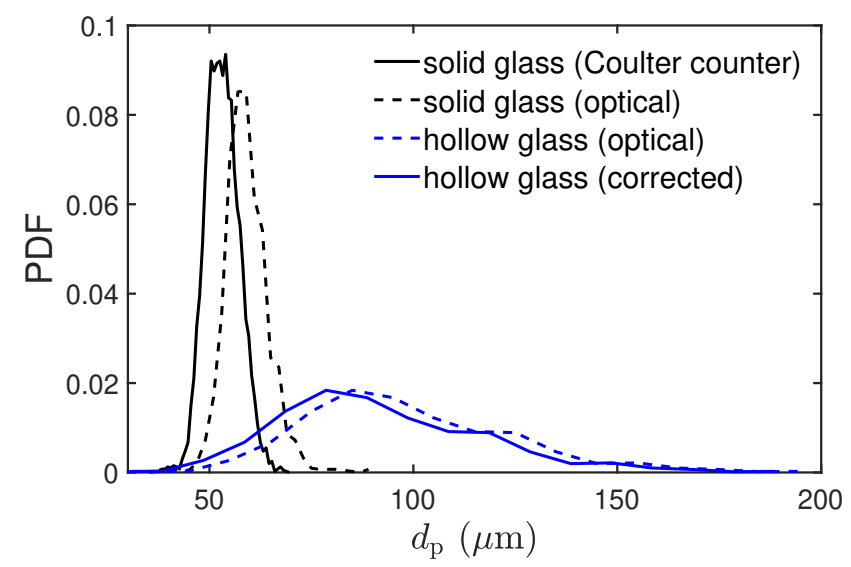

Figure 2: Distribution of particle diameters as measured by Coulter counter and high-resolution imaging.

The hollow glass particles cannot be submerged in the electrolytic solution, because their average specific gravity is less than one, so the Coulter counter is a poor choice for sizing these particles. Alternatively, we use high-resolution static imaging of the particles on a diffuse back-lit surface to obtain a mean diameter of $98 \mu \mathrm{m}$ and a standard deviation of $26 \mu \mathrm{m}$. The bias of the optical size distribution measurement is evaluated by also imaging the solid glass particles and comparing with the corresponding Coulter counter data. The PDFs of the solid glass particles are found to have similar shapes, but the optical measurement yields a PDF that is offset toward larger particle diameters by about $6 \mu \mathrm{m}$. This is likely due to the point spread function and other sources of aberration from the camera lens, which tend to enlarge the shadow boundary of the particles in the images. The final hollow glass size distribution after applying a $6 \mu \mathrm{m}$ offset correction is plotted in solid blue in Fig. 2, resulting in a mean diameter of $92 \mu \mathrm{m}$.

The particle densities $\rho_{\mathrm{p}}$ are measured using the liquid pycnometry method. By mixing a known mass of particles with water and measuring the total slurry volume and mass, the average particle density is inferred. The densities of the solid and hollow glass particles are found to be $2450 \mathrm{~kg} / \mathrm{m}^{3}$ and $136 \mathrm{~kg} / \mathrm{m}^{3}$ respectively.

The particle response time $\tau_{\mathrm{p}}$ relative to the fluid time scale $\tau_{\mathrm{f}}$ is quantified by the Stokes number $S t \equiv$ $\tau_{\mathrm{p}} / \tau_{\mathrm{f}}$ and governs the behavior of the dispersed phase. When $\tau_{\eta}$ is used as the fluid time scale, the degree of preferential concentration reaches a maximum around $S t \approx 1$. The Schiller-Naumann drag correlation is used in Eq. 2 for defining the particle time response,

$$
\tau_{\mathrm{p}}=\frac{\rho_{\mathrm{p}} d_{\mathrm{p}}^{2}}{18 \mu} \frac{1}{1+0.150 R e_{\mathrm{p}}^{0.687}},
$$

where $\mu$ is the fluid dynamic viscosity and $R e_{\mathrm{p}}=u^{\prime} d_{\mathrm{p}} / v$ is the particle Reynolds number. The particle settling velocity $u_{\mathrm{t}}$ is less than $u^{\prime}$, so $u^{\prime}$ is the most appropriate choice of velocity scale in the particle 
Reynolds number definition. A summary of the important average quantities for each set of particles is given in Table 2 .

Table 2: Average particle properties.

\begin{tabular}{cccccc}
\hline & $d_{\mathrm{p}}(\mu \mathrm{m})$ & $\rho_{\mathrm{p}}\left(\mathrm{kg} / \mathrm{m}^{3}\right)$ & $u_{\mathrm{t}}(\mathrm{m} / \mathrm{s})$ & $R e_{\mathrm{p}}$ & $S t$ \\
\hline Solid glass & 53 & 2450 & 0.19 & 2.6 & 14 \\
Hollow glass & 92 & 136 & 0.035 & 4.5 & 2.1 \\
\hline
\end{tabular}

\subsection{Particle tracking}

The particles are tracked over two consecutive frames using both monoscopic and stereoscopic camera setups. For the mono-PTV experiments, particle images are recorded on a single TSI model 630094 CCD camera with a $6600 \times 4400$ pixel array. An AF Micro-Nikkor $200 \mathrm{~mm}$ lens with $f / 8$ aperture setting results in an image magnification of 0.74 . For the stereo-PTV experiments, a pair of TSI model 630159 cameras, each with a $2048 \times 2048$ pixel array, are used. The stereo cameras are configured at a $76^{\circ}$ opening angle and are equipped with $135 \mathrm{~mm}$ lenses at a $15^{\circ}$ tilt angle to achieve coplanar focus. Table 3 gives a complete summary of the PTV parameters.

The particles are illuminated by a New Wave Solo III-15 dual-pulse Nd:YAG laser with a wavelength of $532 \mathrm{~nm}$. The beam passes through a set of lenses to produce a laser sheet of constant height. The $1 / e^{2}$ laser sheet thickness is found to be $1.2 \mathrm{~mm}$ using the knife-edge traverse method, with $\pm 6.5 \%$ spatial deviation across the field-of-view. Variation in the laser intensity in space can cause a non-uniformity in the number of particles detected and bias the resulting particle statistics. To better quantify the degree of laser uniformity, we compute the particle concentration field, in particles per unit area, as recorded on the mono camera. The average concentration of solid glass particles is found to be $14.7 \mathrm{~cm}^{-2}$, with maximum spatial deviation of $\pm 10 \%$. The inter-frame time $\delta t$ is set to $100 \mu$ s for the mono-PTV experiment and $300 \mu$ s for the stereo-PTV experiment, which results in an average particle displacement of about 8 pixels. At least 2000 image pairs are collected in each experiment to achieve statistical convergence.

Particle coordinates are determined by thresholding the background subtracted images and extracting the centroid of each identified region. In the stereo-PTV experiments, the image plane coordinates are mapped to physical space using the calibration procedure outlined in Machicoane et al.(2019). Briefly, each centroid detected on a camera corresponds to a ray path that passes through the measurement volume. Stereo pairing is achieved when the minimum distance between two ray paths from unique cameras falls below a chosen threshold. The corresponding particle coordinate is then identified as the midpoint of the line that is normal to both ray paths. A given ray path is allowed to have more than one stereo pairing, in order to reduce the effect of particle overlap in either of the stereo camera views. Although entirely possible, it is unlikely that

Table 3: List of PTV parameters for mono and stereo experiments.

\begin{tabular}{lcc}
\hline & Mono-PTV & Stereo-PTV \\
\hline Laser sheet thickness, $\delta z$ & $1.2 \mathrm{~mm}$ & $1.2 \mathrm{~mm}$ \\
Inter-frame time, $\delta t$ & $100 \mu \mathrm{s}$ & $300 \mu \mathrm{s}$ \\
Repetition time & $0.69 \mathrm{~s}$ & $0.69 \mathrm{~s}$ \\
Lens focal length & $200 \mathrm{~mm}$ & $135 \mathrm{~mm}$ \\
Lens aperture & $f / 8$ & $f / 8$ \\
Camera resolution & $7.4 \mu \mathrm{m} / \mathrm{pixel}$ & $\approx 21 \mu \mathrm{m} / \mathrm{pixel}$ \\
Field of view & $49.1 \times 32.7 \mathrm{~mm}$ & $50.0 \times 37.5 \mathrm{~mm}$ \\
Image pairs acquired & 2350 & 2000 \\
\hline
\end{tabular}


overlapping would occur for a single particle in both camera views simultaneously, given the large camera opening angle.

Once particle coordinates are identified, particles are linked between frame pairs using the nearest neighbor approach described in Ouellette et al. (2006). Conflicting links can arise when a particle in the second frame is the nearest neighbor for more than one particle in the first frame. When this is the case, the links are chosen such that the sum of all particle displacements is minimized.

\section{Results}

\subsection{Radial distribution function}

The RDF $g(r)$ is the first statistical quantity needed to compute the collision rate using Eq. 1. It quantifies the likelihood of finding a particle a distance between $r$ and $r+d r$ from any reference particle relative to the case of uniform random particle distribution. When a measurement of the RDF is carried out, it is important to distinguish the three-dimensional RDF $g_{3}(r)=g(r)$ from lower-dimensional RDFs, such as the two-dimensional RDF $g_{2}(r)$, which deviates from $g(r)$ when $r<\delta z$. We follow the algorithmic procedure for computing the $n$-dimensional RDF described by Larsen and Shaw (2018), which is reflected by

$$
g_{n}(r)=\frac{\sum_{i=1}^{N_{\mathrm{p}}} P_{i}(r) / \delta V_{i}(r)}{\left(N_{\mathrm{p}}-1\right) N_{\mathrm{p}} / V} .
$$

In Eq. 3. $P_{i}(r)$ is the number of particle pairs separated from the $i^{\text {th }}$ particle by a distance $r$ and $r+d r, \delta V_{i}(r)$ is the shell volume in $n$-dimensions between radii $r$ and $r+d r, N_{\mathrm{p}}$ is the total number of particles, and $V$ is the total measurement volume. For the $S t=14$ particles, $g_{2}(r)$ is computed directly from the mono-PTV data. Although a separate mono-PTV experiment was not conducted for the $S t=2.1$ particles, $g_{2}(r)$ is emulated by projecting the stereoscopic particle coordinates onto the $x_{1}-x_{2}$ plane and then computing Eq. 3 .
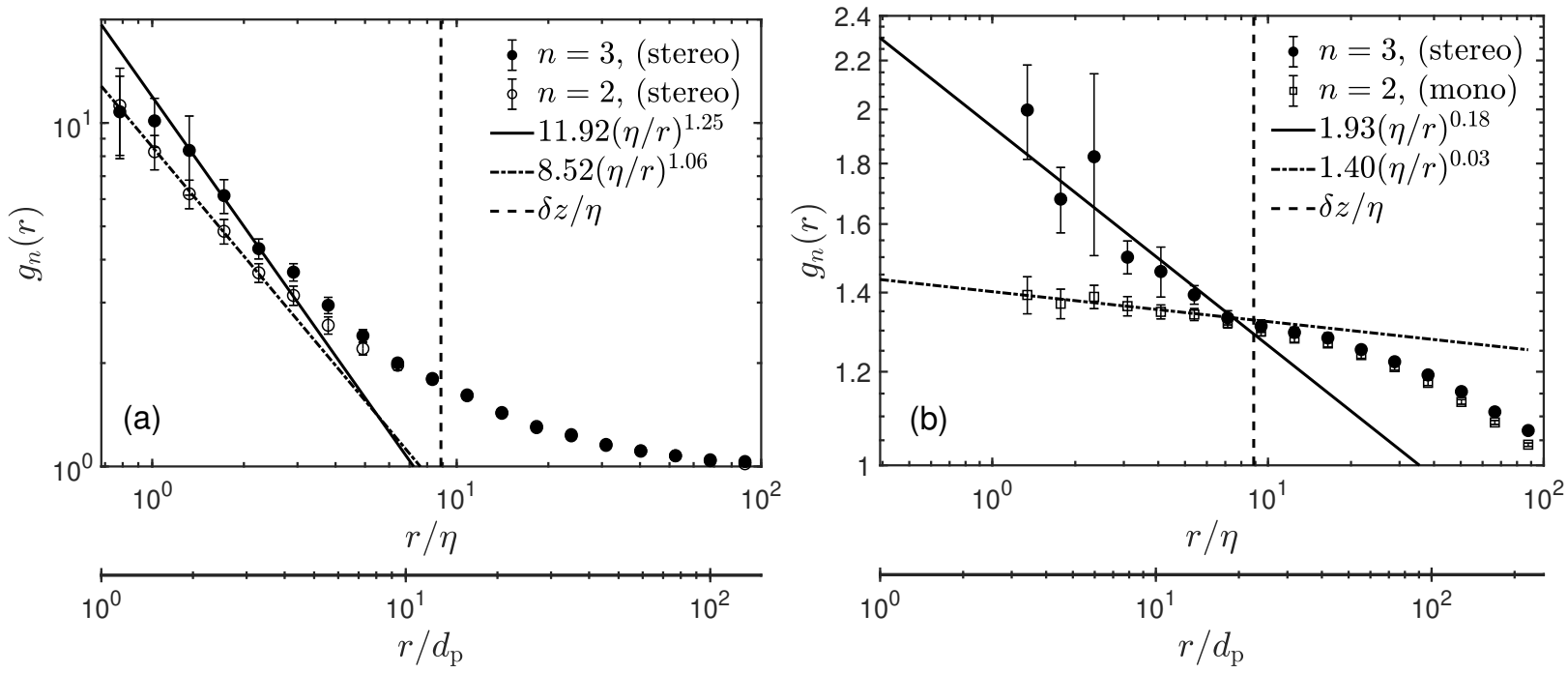

Figure 3: Radial distribution function $g_{n}(r)$ computed from two-dimensional $(n=2)$ and three-dimensional $(n=3)$ particle coordinates for (a) $S t=2.1$ and (b) $S t=14$.

In Fig. 3, $g_{2}(r)$ and $g_{3}(r)$ are plotted for $S t=2.1$ (left) and $S t=14$ (right). To the right of $r=$ $\delta z$, indicated by the vertical dashed line, $g_{2}(r)$ and $g_{3}(r)$ collapse to a single curve. For $r<\delta z, g_{2}(r)$ deviates significantly from the true RDF. The attenuation of $g_{2}(r)$ stems from the fact that particle separation distances are aliased from larger to smaller values when projected onto a plane from a 3D volume, as described by Holtzer and Collins (2002). This bias increases as particles move closer together. Because 
Eq. 1 depends on the RDF at particle contact $g\left(d_{\mathrm{p}}\right)$, and $\delta z \gg d_{\mathrm{p}}$, the two-dimensional RDF provides an underestimate of $N_{\mathrm{c}}$.

The stereo-PTV experiments provide 3D particle coordinates, so the plots of $g_{3}(r)$ in Fig. 3 are free from projection errors and are the appropriate values to use in predicting $N_{\mathrm{c}}$. However, the RDF could only be measured accurately for $r \gtrsim 2 d_{\mathrm{p}}$, due to diffraction effects when imaging the particles, so a functional form is required for extrapolation to $r=d_{\mathrm{p}}$. Reade and Collins (2000) proposed a power-law fit for small $r$, given by Eq. 4, which showed good agreement with RDFs attained from point particle simulations in the range $0 \leq S t \leq 4$.

$$
g(r)=c_{0}\left(\frac{\eta}{r}\right)^{c_{1}}
$$

In the $S t=2.1$ experiments, power-law behavior is observed for $r / \eta<3$. In the $S t=14$ experiments, power-law behavior appears to persist toward larger values, up to $r / \eta \approx 8$. The coefficients $c_{0}$ and $c_{1}$ are obtained for both $g_{2}(r)$ and $g_{3}(r)$ by fitting Eq. 4 over the applicable range of $r$ and are listed in Table 4 .

Table 4: Power-law fitting parameters describing the measured RDFs for small $r$.

\begin{tabular}{ccccccccc}
\hline & \multicolumn{3}{c}{$S t=2.1$} & & \multicolumn{3}{c}{$S t=14$} \\
\cline { 2 - 3 } \cline { 6 - 8 } & $c_{0}$ & $c_{1}$ & $g\left(\bar{d}_{\mathrm{p}}\right)$ & & $c_{0}$ & $c_{1}$ & $g\left(\bar{d}_{\mathrm{p}}\right)$ \\
\hline$g_{3}(r)$ & 11.92 & 1.25 & 19.3 & & 1.93 & 0.18 & 2.28 \\
$g_{2}(r)$ & 8.52 & 1.06 & 12.8 & & 1.40 & 0.03 & 1.44 \\
Holtzer \& Collins fit & 29.29 & 1.81 & 58.6 & & 1.47 & 0.05 & 1.54 \\
\hline
\end{tabular}

For comparison, an alternate approach commonly used to estimate $g\left(d_{\mathrm{p}}\right)$ is to compute $g_{2}(r)$ from monoPTV data and apply a fitting procedure proposed by Holtzer and Collins (2002). Their correction results in two coefficients identical to those used in Eq. 4 in order to recover the true unattenuated RDF. The method relies on the assumption that $g_{2}(r)$ derives from a power-law RDF (at small $r$ ), which is confirmed here by examination of the stereo-PTV data. Holtzer and Collins showed that the correction recovers the power-law coefficients of the true RDF to within $10 \%$ when $\delta z / \eta<4.90$. However, in our experiments, $\delta z / \eta=8.9$, so the errors are expected to exceed $10 \%$. The coefficients obtained using their fit are listed in Table 4 . While the coefficients are adjusted in the right direction when compared to those obtained from the direct fits to $g_{2}(r)$, they are overpredicted for $S t=2.1$ and underpredicted for $S t=14$ when compared to the coefficients obtained from the direct fits to $g_{3}(r)$. It appears that for a large degree of preferential concentration and $\delta z / \eta \sim 10$, the Holtzer and Collins fitting procedure performs worse than a direct power-law fit to $g_{2}(r)$.

\subsection{Relative velocity distribution}

The second statistic needed to compute the collision rate from Eq. 1 is the mean approach velocity, defined by

$$
\left\langle w_{\mathrm{r}, n}\right\rangle^{(-)}(r)=\int_{-\infty}^{0}-w_{\mathrm{r}, n} P\left(w_{\mathrm{r}, n} \mid r\right) d w_{\mathrm{r}, n},
$$

where $P\left(w_{\mathrm{r}, n} \mid r\right)$ is the PDF of radial relative velocity computed in $n$-dimensions conditioned on particle separation distance $r$. The two-dimensional velocity $w_{\mathrm{r}, 2}$ and the three-dimensional velocity $w_{\mathrm{r}, 3}$ are sampled from more than half a million particle pairs in the mono and stereo-PTV datasets respectively. A single sample is extracted from each particle pair by first projecting the pair of lab-frame velocities onto the radial line that connects their centroids and then taking the difference. Finally, the samples are binned according to the particle pair separation distance.

The PDFs of $w_{\mathrm{r}, n}$ are plotted in Fig. 4 at two chosen separation distances, $r / \eta=5.8$ and $r / \eta=23$. The $\mathrm{PDF}$ for $S t=0$ particles is also given as a reference, which are extracted from the instantaneous PIV velocity 

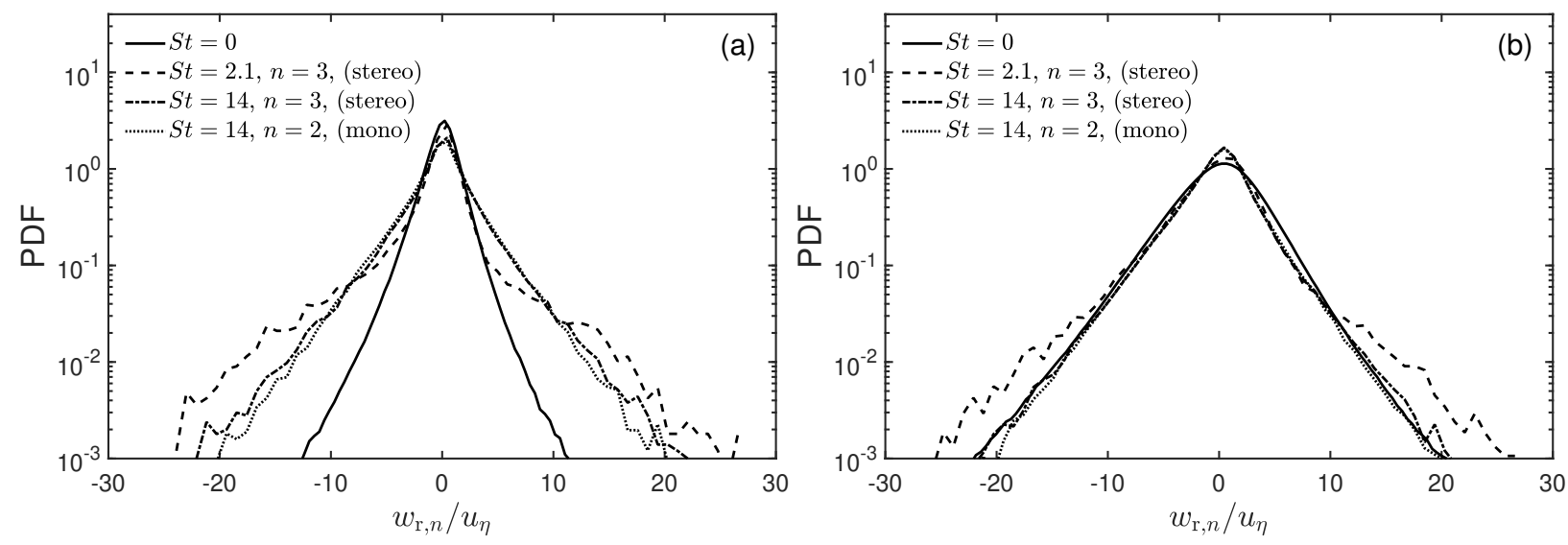

Figure 4: PDFs of normalized radial relative velocity $w_{\mathrm{r}, n} / u_{\eta}$ from two-dimensional $(n=2)$ and threedimensional $(n=3)$ measurements for (a) $r / \eta=5.8$ and (b) $r / \eta=23$.

fields obtained using tracer particles in the experiments conducted by Hoffman and Eaton (2021). The distribution for the inertial particles is wider than the $S t=0$ distribution at $r / \eta=5.8$ but somewhat narrower in the center of the distribution at $r / \eta=23$. This demonstrates the "sling" effect (Falkovich and Pumir, 2007), or caustics, which occurs at small scales of separation, and the filtering effect (Ayyalasomayajula et al., 2008), which occurs at larger scales of separation. The distribution of velocities for $S t=14$ appears to be independent of the measurement type, since the mono and stereo-PTV results are nearly identical, ignoring statistical noise. However, deviations can be expected in the distributions for $r<\delta z$. At these small separations, projection errors in the mono-PTV measurement can cause small relative velocities to alias toward larger velocities, resulting in heavier tails in the distribution. However, there are too few particle pairs contained in the smaller radial bins to display meaningful PDFs. The relative velocity distribution for the $S t=2.1$ particles more closely resemble $S t=0$ particles near the center of the distribution. However, the distribution tails are significantly thicker than expected, and could be a result of frame-pair mismatching introduced by the nearest neighbor tracking algorithm.

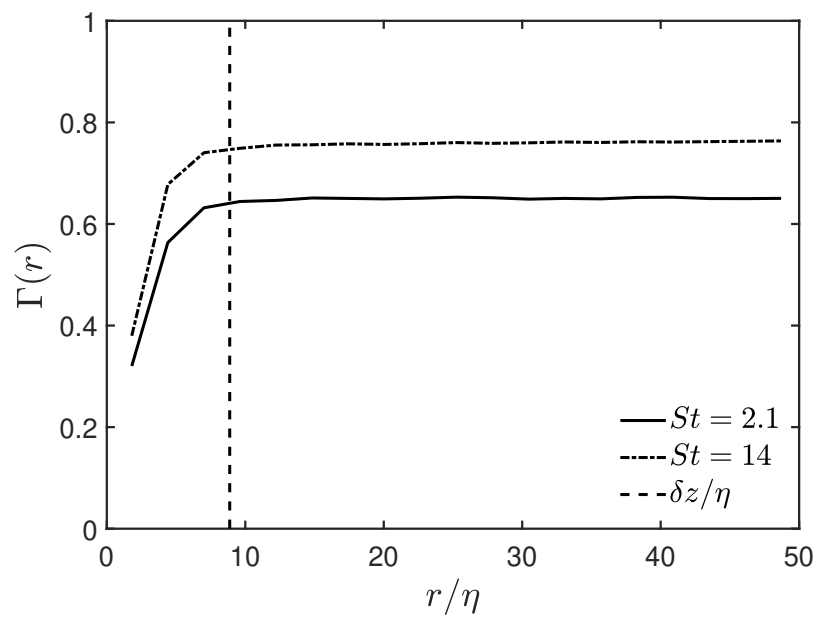

Figure 5: Nearest neighbor yield parameter $\Gamma$ as a function of $r / \eta$ from the stereo-PTV experiments.

A potential source of bias in the radial relative velocity measurement is quantified by comparing the number of particles identified in the first frame of all PTV frame pairs with the number of particles that are successfully matched after applying the nearest neighbor algorithm between frame pairs. In practice, there is always some loss of particles in the second frame due to the finite laser sheet thickness and strong out-of-plane motion. It is useful then to define a yield parameter, 


$$
\Gamma(r)=\frac{\sum_{i=1}^{N_{\mathrm{p}}^{(1)}} P_{i}^{(1)}(r)}{\sum_{i=1}^{N_{\mathrm{p}}^{(1-2)}} P_{i}^{(1-2)}(r)},
$$

where $N_{\mathrm{p}}^{(1)}$ is the total number of particles identified in the first frame of the image pairs, $P_{i}^{(1)}(r)$ is the number of particle pairs in the first frame separated from the $i^{\text {th }}$ particle by a distance $r$ and $r+d r, N_{\mathrm{p}}^{(1-2)}$ is the total number of particles that are successfully matched using the nearest neighbors algorithm, and $P_{i}^{(1-2)}(r)$ is the number of successfully matched particle pairs separated from the $i^{\text {th }}$ particle by a distance $r$ and $r+d r$. To summarize, $\Gamma$ quantifies the fraction of particles in the first frame that are successfully matched in the second frame conditioned on $r$. In Fig. 5, $\Gamma$ is plotted against the normalized separation distance $r / \eta$. It appears that $\Gamma$ is not a function of $r$ when $r / \eta>10$, reaching a plateau of 0.65 for $S t=2.1$ and 0.76 for $S t=14$. The lower yield for the $S t=2.1$ particles overall is likely due to greater out-of-plane motion, since the particle rms velocity is larger.

Particles within a region of strong preferential concentration are generally more difficult to track with high fidelity, which partly explains the decline in yield for nearby particles. More importantly, the laser sheet thickness is $\delta z / \eta=8.9$, which corresponds to the point at which $\Gamma$ falls off. This suggests that overlap of nearby particles in the images is quite common. If two nearby particles are identified in the first frame, there is some probability that their images will overlap in at least one camera view of the second frame. If the particle pair has an inward radial relative velocity, then the likelihood of overlap is further increased. If this is the case, fewer samples are collected when $w_{\mathrm{r}, 3}$ is negative, causing a skew in the relative velocity distribution toward positive values at small $r$.
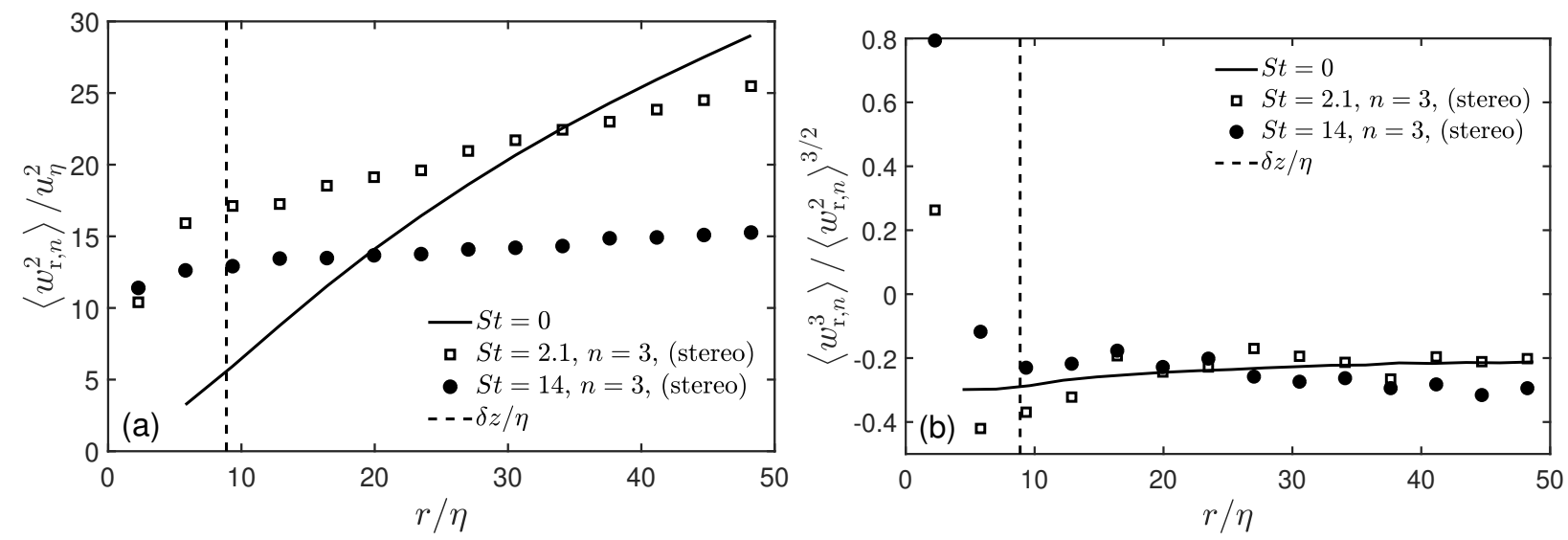

Figure 6: (a) Normalized variance and (b) skewness of the relative velocity distributions as a function of particle separation for $S t-2.1$ and $S t=14$ as measured by stereo-PTV. The $S t=0$ data is taken from 2D PIV data.

The shape of the relative velocity distributions can be better understood over all separation distances by plotting the normalized variance $\left\langle w_{\mathrm{r}, n}^{2}\right\rangle / u_{\eta}^{2}$ and the skewness $\left\langle w_{\mathrm{r}, n}^{3}\right\rangle /\left\langle w_{\mathrm{r}, n}^{2}\right\rangle^{3 / 2}$ as functions of $r$. The variance, given in Fig. 6 a , highlights the steady decrease in the correlation of particle motion as the distance separating them increases. The distribution skewness, shown in Fig. 6b, takes mostly negative values and exhibits minor deviations from the $S t=0$ trend computed from the PIV fields. It is important to point out that two-point relative velocity statistics in turbulent flows are inherently asymmetric, as reported by Tavoularis et al. (1978). Particles can have more asymmetric relative velocity distributions than the underlying flow, due to their inertia, with the skewness reaching its most negative value for $S t \approx 1$ (Ray and Collins, 2011). For this reason, skewness has often been linked to preferential concentration. From Fig. 6h, we observe that the skewness increases slightly with $r$ for $S t=2.1$ and decreases with $r$ for $S t=14$. These trends were also observed in the numerical study of Ray and Collins (2011), although the magnitude of skewness 
reported there was several times higher. Two possible explanations for this could be the higher $R e_{\lambda}$ in our experiments and the effect of polydispersity. The positive skewness observed on the left-most side of Fig. 6 is an artifact of the nearest neighbor algorithm, and is only shown here to indicate the limitation of the measurement in capturing the radial relative velocity statistics.

To obtain the mean approach velocity $\left\langle w_{\mathrm{r}, n}\right\rangle^{(-)}(r)$, all radial relative velocity samples in each radial bin that are less than zero are averaged together. In Fig. 7, the mean approach velocity is plotted as a function of particle separation for both particle Stokes numbers. The solid black line indicates the mean approach velocity for $S t=0$ particles, which is computed from the instantaneous PIV velocity fields (Hoffman and Eaton, 2021). As expected, the mean approach velocity increases monotonically with $r$.
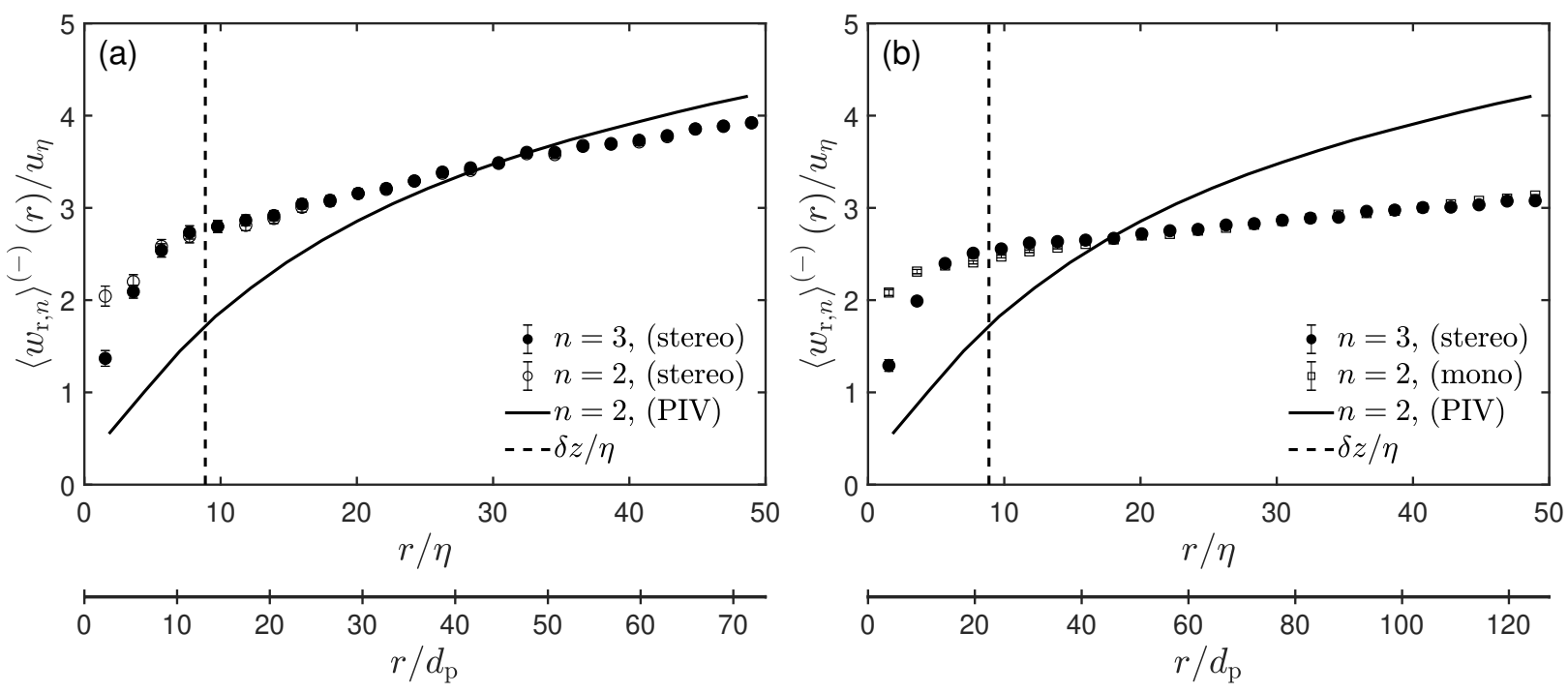

Figure 7: Mean approach velocity computed from two-dimensional $(n=2)$ and three-dimensional $(n=3)$ data for (a) $S t=2.1$ and (b) $S t=14$.

To the right of the vertical dashed line, which indicates the laser sheet thickness $r=\delta z$, the results are the same regardless of whether two or three-dimensional velocities are used. For $r<\delta z$, the mean approach velocity computed from the two-dimensional data is augmented relative to the three-dimensional data due to the projection errors. The smallest separation at which $\left\langle w_{\mathrm{r}, n}\right\rangle^{(-)}(r)$ can be reliably obtained from the three-dimensional measurement is $r / \eta \approx 5$. This limiting value arises from the artificial positive skewness of the relative velocity distribution seen in Fig. 6b, which occurs for $r / \eta \lesssim 5$. Because the two and threedimensional measurements align closely above this lower limit, stereo-PTV offers little additional advantage over mono-PTV in estimating the mean approach velocity at particle contact.

\section{Conclusions}

Two important particle pair statistics relevant for inter-particle collisions, the RDF and the mean approach velocity, are measured for two types of particles having an average Stokes number of 2.1 and 14, and diameters of $0.68 \eta$ and $0.39 \eta$ respectively. Stereo-PTV is used to estimate these quantities at separations near particle contact. The RDFs, which are obtained down to $r \approx \eta$, exhibit power-law behavior at small separations, even for the higher Stokes number. At small separation, the RDF varies by nearly an order of magnitude over the range of $S t$ measured. The advantage of using stereo-PTV over a two-dimensional technique is clear, given that the RDFs at particle contact are underpredicted by about a factor of 2 when mono-PTV data is used. Thus, a three-dimensional measurement technique like stereo-PTV is necessary to obtain accurate estimates of the volumetric collision rate. Adding a third or fourth camera view can incrementally boost the accuracy of the RDF measurement, with the cost of additional complexity in calibration, measurement synchronization, and data analysis. For applications where a two-dimensional particle detection technique must be used, the Holtzer and Collins power-law fitting procedure may not be reliable when the dimensionless laser sheet thickness $\delta z / \eta$ is greater than 4.9. 
The mean approach velocity is also obtained at small particle separation, but is found to be reliable only down to $r / \eta \approx 5$. This limiting value for $r$ is based on the location of nonuniformity in the yield parameter $\Gamma(r)$ as well as the anomalous skewness in the radial relative velocity distributions. Two additional approaches could be used in the future to overcome these limitations: (1) counteract particle overlap in images through additional stereo camera views, and (2) improve position and velocity certainty over the particle tracks through continuous (high-speed) particle tracking.

\section{Acknowledgements}

This material is based upon work supported by the National Science Foundation under Grant EAR-1756068. The code used to generate links defining the particle tracks was generously provided by N. Ouellette.

\section{References}

Ayyalasomayajula S, Warhaft Z, and Collins LR (2008) Modeling inertial particle acceleration statistics in isotropic turbulence. Physics of Fluids 20:095104

De Jong J, Salazar JP, Woodward SH, Collins LR, and Meng H (2010) Measurement of inertial particle clustering and relative velocity statistics in isotropic turbulence using holographic imaging. International Journal of Multiphase Flow 36:324-332

Dou Z, Ireland PJ, Bragg AD, Liang Z, Collins LR, and Meng H (2018) Particle-pair relative velocity measurement in high-Reynolds-number homogeneous and isotropic turbulence using 4-frame particle tracking velocimetry. Experiments in Fluids 59:30

Eaton JK and Fessler JR (1994) Preferential concentration of particles by turbulence. International Journal of Multiphase Flow 20:169-209

Falkovich G and Pumir A (2007) Sling effect in collisions of water droplets in turbulent clouds. Journal of the Atmospheric Sciences 64:4497-4505

Hoffman DW and Eaton JK (2021) Isotropic turbulence apparatus with a large vertical extent. Manuscript submitted for publication

Holtzer GL and Collins LR (2002) Relationship between the intrinsic radial distribution function for an isotropic field of particles and lower-dimensional measurements. Journal of Fluid Mechanics 459:93-102

Larsen ML and Shaw RA (2018) A method for computing the three-dimensional radial distribution function of cloud particles from holographic images. Atmospheric Measurement Techniques 11:4261-4272

Machicoane N, Aliseda A, Volk R, and Bourgoin M (2019) A simplified and versatile calibration method for multi-camera optical systems in 3D particle imaging. Review of Scientific Instruments 90:35112

Ouellette NT, Xu H, and Bodenschatz E (2006) A quantitative study of three-dimensional Lagrangian particle tracking algorithms. Experiments in Fluids 40:301-313

Ray B and Collins LR (2011) Preferential concentration and relative velocity statistics of inertial particles in Navier-Stokes turbulence with and without filtering. Journal of Fluid Mechanics 680:488-510

Reade WC and Collins LR (2000) Effect of preferential concentration on turbulent collision rates. Physics of Fluids 12:2530-2540

Sundaram S and Collins LR (1997) Collision statistics in an isotropic particle-laden turbulent suspension. Part 1. Direct numerical simulations. Journal of Fluid Mechanics 335:75-109

Tavoularis S, Bennett JC, and Corrsin S (1978) Velocity-derivative skewness in small Reynolds number, nearly isotropic turbulence. Journal of Fluid Mechanics 88:63-69 